\title{
FUNCIONALIDADE DAS APLICAÇÕES DOS DISPOSITIVOS IOS E MAC VIA ICLOUD
}

\section{FUNCTIONALITY OF THE APPLICATION OF IOS AND MAC DEVICES VIA ICLOUD}

\author{
Lucas Hans Ramos ${ }^{1}$;Eliane Vendramini de Oliveira ${ }^{2}$ \\ ${ }^{1}$ Graduando em Análise e Desenvolvimento de Sistemas/FATEC-Presidente Prudente; \\ 2Docente/ FATEC-Presidente Prudente e FAI-Faculdades Adamantinenses Integradas. \\ e-mail: lucashansg@gmail.com
}

RESUMO - O presente trabalho aborda a funcionalidade das aplicações em dispositivos móveis e desktops utilizando-se a tecnologia iCloud voltada para dispositivos iOS e Mac. O trabalho como um todo se baseia na Worldwide Developers Conference, que é onde desenvolvedores da Apple Inc. se reúnem para mostrar os avanços feitos para aquele determinado ano corrente. O trabalho ainda apresenta em seu conteúdo aspectos da Inteligência Artificial com a utilização do iCloud. O desenvolvimento do trabalho se mostra como um manual de grande utilidade para usuários no Brasil interessados em conhecer um pouco a respeito da tecnologia iCloud e suas aplicações. Este trabalho acadêmico se torna de grande ajuda e interesse, pois além de ser baseado na WWDC, se baseia em grandes fontes de pesquisa bem referenciadas, como o website 9to5Mac, a maior fonte atual de informações referentes a dispositivos APPLE e a revista tecnológica americana THE VERGE, na qual o conteúdo referente sobre IA esta sendo quase inteiramente baseado.

Palavras-chave: WWDC; Apple; iCloud; Apps; Inteligência Artificial.

\begin{abstract}
This paper discusses the functionality of applications on mobile and desktop devices using the iCloud technology targeted for iOS and Mac devices. The work as a whole is based at the Worldwide Developers Conference, which is where developers have a meeting at Apple Inc. to show the advances made for that year. The work also shows in its content aspects of Artificial Intelligence using iCloud. The development work is shown as an useful manual for users mostly in Brazil interested in knowing a little about iCloud technology and its applications. This academic work becomes of a great help and interest, as well as being based at WWDC, is based in large research sources as well as the referenced website 9to5Mac, the most current source of information for APPLE devices, and the technological American magazine THE VERGE, which almost all the content about Artificial Intelligence is being based on it.
\end{abstract}

Keywords: WWDC; Apple; iCloud; Apps; Artificial Intelligence. 


\section{INTRODUÇÃO}

A palavra nuvem sugere uma ideia de ambiente desconhecido, o qual se pode ver somente seu início e fim. Por este motivo esta foi muito bem empregada na nomenclatura deste novo modelo, onde toda a infraestrutura e recursos computacionais ficam "escondidos", tendo o usuário o aceso apenas a uma interface padrão através da qual é disponibilizado todo o conjunto de variadas aplicações e serviços.

A nuvem é representada pela Internet, isto é, a infraestrutura de comunicação composta por um conjunto de hardwares, softwares, interface, redes de telecomunicação, dispositivos de controle e de armazenamento que permitem a entrega da computação como serviço.

Para tornar este modelo possível, é necessário reunir todas as aplicações e dados dos usuários em grandes centros de armazenamento, conhecidos como data centers. Uma vez reunidos, a infraestrutura e as aplicações dos usuários são distribuídas na forma de serviços disponibilizados por meio da internet.

Outro ponto importante para o entendimento deste modelo de computação refere-se aos participantes da nuvem. Estes podem ser divididos em três grandes grupos: Provedor de serviço, desenvolvedor e usuário.
De acordo com Pedrosa, Paulo H.C e Tiago Nogueira (Online, 2014).

O provedor, responsável pela tarefa de disponibilizar gerenciar e monitorar toda a infraestrutura da nuvem garante o nível do serviço e a segurança adequada de dados e aplicações. Já o desenvolvedor deve ser capaz de prover serviços para o usuário final, a partir da infraestrutura disponibilizada pelo provedor de serviço. Enquanto o usuário final é o consumidor que irá utilizar os recursos oferecidos pela nuvem computacional.

A computação nas nuvem representa um novo modelo de serviço capaz de fornecer todo o tipo de processamento, infraestrutura e armazenamento de dados através da internet, baseado na necessidade do usuário.

Hoje, o mundo moderno é voltado para as facilidades em que o compõem, todos querem que métodos, que ontem eram complexos, que hoje se tornem mais fáceis. Antigamente utilizava-se o disco como principal componente para 0 armazenamento de músicas. Um pouco mais adiante foi evoluindo cada vez mais, até o surgimento do $C D$. As pessoas simplesmente amavam essa nova forma de armazenamento, pois podiam escutar suas músicas favoritas no momento que elas quisessem. Diante dessa necessidade, criam- 
se inúmeras formas de armazenamentos, e cada vez com maior capacidade de armazenamento. Mas eis que surge uma questão. Muitos podem-se perguntar o como fazer para que se possa alterar um arquivo, mas este arquivo não esteja fisicamente no HD, e que se possa utilizá-lo em tempo real.

A ideia que se teve é a de colocar tudo em nuvem, e fazer com que os usuários consigam se conectar. Desenvolvedores ao elaborarem a idéia de iCloud, já tinham em mente uma direção correta. Eles já sabiam o quão grande essa pequena ideia traria com aplicações e funcionalidades para os sistemas iOS e Mac.

Hoje no Brasil, protótipos Apple tais como, iPhones, iPods, iPads, Macs, entre outros, ainda são muito caros, o que torna-os pouco conhecidos na prática. Esta pesquisa justifica-se como um manual que almeja difundir mais este assunto para todos aqueles que desejam possuir tal dispositivo, mas que não sabem como tal funciona. Embora, dispositivos iOS e Mac possuem vasta quantidade de materiais para pesquisa, iCloud não possui, por ser uma tecnologia muito recente ainda. $O$ trabalho justifica-se ainda por ser em quase sua totalidade baseado na WWDC, uma vez que as conferências (Keynotes) são em sua língua oficial, o inglês.

\section{METODOLOGIA}

A metodologia utilizada neste artigo é com base no estudo dos dispositivos iOS e Mac via iCloud, mostrando uma visão geral de suas principais aplicações. O material formado com os estudos foi baseado na WWDC - WORLDWIDE DEVELOPERS CONFERENCE. Portanto se trata de uma de uma pesquisa baseada em conteúdos já existentes.

Este trabalho acadêmico é de pesquisa aplicada, pois objetiva gerar conhecimentos para a aplicação prática dirigido à solução de problemas específicos. A pesquisa denomina-se qualitativa, pois utiliza fatos reais e é descritiva. Visa-se como objetivo uma pesquisa explicativa, pois identifica os fatores que determinam ou contribuem para a ocorrência dos fenômenos. Designa este trabalho acadêmico como uma pesquisa Expost-Facto, pois ocorre quando o experimento se realiza depois dos fatos ocorridos.

\section{RESULTADOS}

Com base nos dispositivos móveis via iCloud, apontam-se conceitos gerais a respeito de informar e esclarecer possíveis dúvidas relacionadas a dispositivos iOS e Mac no Brasil. Dentre as diversas funcionalidades de aplicações entre Mac e dispositivos iOS, aborda-se as principais que são: App Store, iTunes Store, Safari e Siri. 
A App Store, apresentada pela primeira vez em 2008 em uma KEYNOTE por Steve Jobs, simplesmente revolucionou a ideia de downloads de aplicações nos protótipos Apple.

Até então, não existia a possibilidade e de usuários baixarem suas aplicações favoritas, como games, livros e aplicações (apps) sobre culinária. Mesmo para grandes desenvolvedores com as ideias de aplicações em dispositivos móveis em mente, isso tudo até então não era possível. Jobs diz que os desenvolvedores vão passar horas utilizando essa nova aplicação.

A App Store é a maior loja virtual de aplicativos da atualidade. Com ela o usuário é capaz de baixar suas aplicações favoritas e utilizá-las através do iCloud. A dúvida que o usuário tem é, como funciona o iCloud nos seus diferentes dispositivos. No iCloud o funcionamento do mesmo ocorre de que o usuário pode baixar uma mesma aplicação entre diferentes dispositivos sem que ele nem mesmo precise relar no outro dispositivo para que sua aplicação esteja naquele dispositivo, quer dizer, através de um ID (identificação) do usuário, sua mesma aplicação vai aparecer em todos os dispositivos Apple que o usuário possuir.

A iTunes Store mostrada pela primeira vez em 2003 em uma KEYNOTE apresentada por Steve Jobs, simplesmente revolucionou a ideia de downloads de musicas nos protótipos Apple.

Até então, não existia nem ao menos a ideia de download de musicas, porque simplesmente não existia essa ideia. Steve Jobs diz "Quando nós decidimos entrar no mercado de musica, decidimos entrar fortes", pois a APPLE não foi a primeira a desenvolver dispositivos voltados para 0 mercado musical.

Quando a Apple lançou sua primeira geração do ipod, ela bateu a incrível marca de 700.000 ipods vendidos "Dados de 2003 da APPLE.COM" e o ipod se tornou o mp3 player mais vendido no mundo. O iTunes até então era apenas uma aplicação para PC e Mac onde se conecta o ipod e as musica que são compradas, podem ser passadas para o ipod.

A iTunes Store é a maior loja virtual de download de músicas, filmes e séries da atualidade. Com ela o usuário é capaz de baixar suas músicas favoritas e utilizá-las através do iCloud. A idéia de iCloud é a mesma da App Store. Nessa aplicação o usuário faz o download de uma música em seu Mac, e com sua autorização, a mesma música aparece em todos seus dispositivos, sem nenhum custo adicional, pois a maioria dos aplicativos e músicas nas lojas virtuais Apple não são gratuitas. 
O funcionamento do iCloud na iTunes

Store é referente a download de músicas, filmes, séries, livros e áudio books.

O Safari, lançado em 23 de junho de 2003, é o navegador web browsing desenvolvido pela Apple Inc. que segundo ela "Safari é o browsing mais popular e fácil de usar do mundo."

Segundo a Apple Inc., Safari é o web Browser mais utilizado em dispositivos móveis, comparando-se os dispositivos iOS com os dispositivos Android e outros.

De acordo com a Apple Store UK, Safari é o melhor browser para se navegar e se usar do mundo.

O conceito de iCloud no Safari é de que é possível manter todas as abas que o usuário possui abertas e atualizadas entre todos os seus dispositivos, quer dizer, o usuário pode começar uma pesquisa em seu iPhone e quando ele(a) ligar seu Mac, a mesma página em que foi iniciada no iPhone continua aberta no Mac, através do iCloud.

Há décadas desenvolvedores tem sonhado em poder conversar com a tecnologia e ela ser hábil ao ponto de fazer algo útil. Mas isso nunca foi possível, porque sempre existiram limitações de capacidade como, por exemplo, dizendo apenas, ligue para um numero, ou toque uma música.

Segundo PHIL (Vice-presidente sênior de vendas da Apple Inc.) "O que realmente queremos é poder conversar com nosso dispositivo, fazer perguntas simples".

O sistema de inteligência artificial de comando de voz para sistemas iOS ou Siri, foi apresentado pela primeira vez em uma KEYNOTE especial em outubro de 2011 na Califórnia, EUA.

Siri está integrada com o iCloud pelo fato de que se pode fazer tudo o que se faz com o aparelho normalmente, mas com comandos de voz. Por exemplo, um usuário pode pedir para que se toque uma música que está em outro dispositivo iOS via iCloud. Resumindo, o iCloud compartilha um comando de voz que é feito em um iPod diretamente para seu Mac.

\section{DISCUSSÃO}

Segundo Tim Cook (CEO da Apple Inc.), a Apple possui cerca 72 milhões de usuários de Mac no mundo, o dobro do que há 5 anos. Cook diz "O iMac é o desktop mais vendido nos EUA, e o MacBook é o notebook com maior número de vendas" destacou, acrescentando que o mercado de Macs dobrou no último ano, enquanto os PCs desktops cresceram 18\%, dados de 2013 da Apple.com.

O Mac ainda é um computador pouco conhecido em escala mundial. O Windows ainda tem um enorme domínio no mercado com cerca de $91.19 \%$ de computadores vendidos, dados de 2013 do TargetHD. 
Por falta de informações em pesquisa na internet, livros e revistas, foi realizado um levantamento de dados com 150 pessoas da cidade de Pres.Venceslau, SP. O público alvo foram alunos de escolas públicas e particulares. As perguntas foram basicamente duas, se o entrevistado possui um Mac ou iOS ou se já utilizou alguma vez utilizou algum deles.

No Brasil, o conhecimento a respeito de dispositivos iOS e Mac é ainda muito remoto segundo os dados, com cerca de 92.6 $\%$ dos entrevistados terem dito que nunca utilizaram nem ao mesmo o Mac e $74.8 \%$ terem dito que nunca utilizaram um dispositivo iOS.

Quando refere-se a aplicações nos dispositivos iOS e Mac via iCloud, os dados são assustadores, com 97.5\% dos entrevistados terem dito que não sabiam o que é iCloud e os $2.5 \%$ terem dito que sabem o que é, mas que não possuem informações mais relevantes a respeito.

A falta de informação no Brasil ainda é muito grande em relação aos computadores e dispositivos móveis Apple. Diante disso, percebe-se que o conhecimento sobre iCloud com as aplicações tanto do Mac quanto dos dispositivos iOS se tornam muito pobres em informação. Com as funcionalidades das aplicações App Store, iTunes Store, Safari e Siri, as principais entre os dispositivos iOS e Mac, esclarece-se mais o assunto ao usuário que possui dúvidas a respeito desse assunto.

\section{CONCLUSÃO}

Após a leitura do leitor(a), este será capaz de compreender melhor o quanto a tecnologia iCloud é importante nos dias atuais, principalmente se ele(a) possui vários dispositivos móveis ou até mesmo desktops e deseja-se armazenar muita quantidade de informações e acessa-las.

Hoje no Brasil, protótipos Apple não são muito populares, e em consequência disso não existe muita informação na língua portuguesa do Brasil. Esse trabalho, que funciona como um manual, possui todo quase todo o seu conteúdo retirado de fontes estrangeiras, o que ajuda ainda mais aquele(a) leitor(a) que não possui conhecimentos na língua inglesa.

Este trabalho tem como conclusão informar melhor os brasileiros que possuem dúvidas e interesses relacionados a dispositivos Apple e sobre o tecnologia iCloud.

\section{REFERÊNCIAS}

APPLE Inc. Worldwide Developers Conference 2007.Disponível em: $<$ https://www.youtube.com/watch?v=t4OEsl OSc_s> Acesso em: 20 FEV. 2014.APPLE Inc. Worldwide Developers Conference 2008.Disponível em:< https://www.youtube.com/watch?v=Zk97Tu 3PY6I >Acesso em: 22 FEV. 2014. 
APPLE Inc. Worldwide Developers Conference 2009.Disponível em:< https://www.youtube.com/watch?v=6vOvdl8 HJ10 >Acesso em: 5 MAR. 2014.

APPLE Inc. Worldwide Developers Conference 2010.Disponível em:< https://www.youtube.com/watch?v=AmXc1 Mjr5J4 >Acesso em: 12 MAR. 2014.

APPLE Inc. Worldwide Developers Conference 2011.Disponível em: <https://www.youtube.com/watch?v=gfj7Ug CMsqs > Acesso em: 14 MAR. 2014.

APPLE Inc. Worldwide Developers Conference 2012.Disponível em: <https://www.youtube.com/watch?v=DSVCg 1dZLo0> Acesso em: 20 MAR. 2014.

APPLE Inc. Worldwide Developers Conference 2013.Disponível em: <https://www.youtube.com/watch?v=4FunX nJQxYU> Acesso em: 20 MAR. 2014.

HURWITZ, Judith; BLOOR, Robin; KAUFMAN, Marcia; HALPER, Fern.Cloud Computing for Dummies; 1.ed Indiana, U.S. : Wiley Publishing, Inc; 2010. 336 p. ISBN: 978-0-47048470-8. Acesso em: 15 MAR. 2014.

Pedrosa, Paulo H. C, Nogueira, Tiago. Computação em Nuvem. Disponível em: <http://www.ic.unicamp.br/ ducatte/mo401 /1s2011/T2/Artigos/G04-095352-120531t2.pdf> Acesso em: 14 MAR. 2014.

Targethd $\mathbf{0}$ Windows no mercado. Disponível em: $<$ http://targethd.net/windows-aindadomina-o-mercado-de-desktops-maswindows-8-nao-passa-de-7-dos-pcs/> Acesso em: 20 MAR. 2014. 